\title{
LEBESGUE SPACES OF SUMMABLE FUNCTIONS ${ }^{1}$
}

\author{
DAVID M. TOPPING
}

1. Introduction. The classical theorem of Kakutani [1] provides an elegant characterization of $L^{1}$ in terms of its lattice order and special properties of the norm. Much of the difficulty in representing an abstract $(L)$-space apparently stems from the fact that the end result must in general be a vector lattice whose elements are Lebesgue classes of functions rather than functions themselves. In this paper we give a characterization of $\mathfrak{L}^{1}$, the space of summable functions. Toward this end, we abstract the essential properties of $\mathscr{L}^{1}$ in the following:

Definition. An (a\&)-space is a vector lattice $V$ with a seminorm $p$ satisfying:

(1) $p(x+y)=p(x)+p(y)$ for $x, y \geqq 0$.

(2) $p(|x|)=p(x)$ for all $x \in V$.

(3) $V$ is complete in the $p$-topology.

(4) There is a total family $\Lambda$ of linear lattice functionals (see $\$ 2$ for definition) such that the subspace

$$
B=\left\{x \in V:\|x\|=\sup _{\lambda \in \Delta}|\lambda(x)|<\infty\right\}
$$

is dense in $V$ under the $p$-topology, and complete under the norm topology given by $\|x\|$.

MAIN Theorem. Let $V$ be an ( $(\mathcal{L})$-space. Then there is a locally compact Hausdorff space $E$ and a unique positive Radon measure $\mu$ on $E$ such that $V$ is linearly, latticially and isometrically isomorphic to $\mathscr{L}^{1}(E, \mu)$, the space of all summable functions on $E$. Of course $V / p^{-1}(0)$ is then abstractly identical with $L^{1}(E, \mu)$.

For example, if we take $V$ to be the space $\mathcal{L}^{1}(X, m)$ of summable functions on a (nontopological) finite measure space $(X, S, m)$ then conditions (1)-(3) are clearly satisfied. For $\Lambda$ we may take the "point measures" (linear lattice functionals) $\epsilon_{a}, a \in X$ where $\epsilon_{a}(f)=f(a)$, for $f$ in $\mathscr{L}^{1}$. $B$ is then the Banach algebra of bounded summable functions and (4) is clearly satisfied.

If we strengthen the above conditions by requiring $p$ to be a norm so that $V$ becomes an $(A L)$-space in Kakutani's sense, then it appears likely that the only such spaces are the $l^{1}$ spaces of summable func-

Received by the editors August 9, 1960.

1 This work was supported in part by an NSF grant to Tulane University and constitutes a portion of the author's doctoral dissertation. 
tions over a discrete space relative to an atomic measure. This question will be considered in a later paper.

2. The auxiliary representation. By a linear lattice functional we mean a linear functional $\lambda: V \rightarrow R$ satisfying $\lambda\left(x^{+}\right)=(\lambda x)^{+}$, for all $x \in V$, where $x^{+}=x \bigvee 0$. Such a functional is evidently positive. Regarding the existence of such functionals we have the following result (Nakayama [3]):

THEOREM 1. Let $V$ be a vector lattice. Then there exists a set $X$ such that $V$ is isomorphic, as a vector lattice, to a pointwise linear sublattice of $R^{X}$ if and only if $V$ has a total family of linear lattice functionals.

Thus condition (4) amounts, in part, to assuming that $V$ is a function lattice. Regarding (4) we observe further that the set $B$ inherits some rather special properties. For a set $\Lambda$, we denote by $m(\Lambda)$ the algebra of all bounded real-valued functions on $\Lambda$.

LEMMA 1. Let $V$ be a pointwise vector lattice of functions on a set $\Lambda$ and let $B=m(\Lambda) \cap V$. Then $B$ is a lattice ideal in $V$, and, a fortiori, an algebra.

Proof. Let $f \in m(\Lambda)$ with $\|f\|>1$ where $\|f\|=\sup \{|f(\lambda)|: \lambda \in \Lambda\}$. Then $\|f\| \leqq\left\|f^{2}\right\|$; for if we take $\lambda \in \Lambda$ so that $|f(\lambda)|>1$, then $|f(\lambda)|$ $<\left|f^{2}(\lambda)\right|$. Again, if $f \in m(\Lambda),\|f\|>1$, then $f^{2} \leqq\left\|f^{2}\right\| \cdot|f|$; for we have $\left\|f^{2}\right\| \cdot|f|-|f|^{2}=\left(\left\|f^{2}\right\|-|f|\right) \cdot|f| \geqq 0$ since $|f| \leqq\|f\| \leqq\left\|f^{2}\right\|$. Now if $g \in B$ and $f \in V$ with $|f| \leqq|g|$, then $\|f\| \leqq\|g\|<\infty$, so $f \in B$, i.e., $B$ is a lattice ideal in $V$. For $0 \neq f \in B$ we choose $0<\epsilon<\|f\|$. Then $g=(1 / \epsilon) f$ has norm $>1$. Hence $0 \leqq g^{2} \leqq\left\|g^{2}\right\| \cdot|g|=\left(\left\|g^{2}\right\| / \epsilon\right) \cdot|f|$ and by the lattice ideal property $g^{2} \in B$. But $f^{2}=\epsilon^{2} g^{2}$, so $f^{2} \in B$. Q.E.D.

Reverting now to Theorem 1 , we map $V$ into $R^{\Delta}$ by $x \rightarrow \bar{x}$ where $\bar{x}(\lambda)=\lambda(x)$ for $x \in V, \lambda \in \Lambda$. The elements of $V$ then appear as functions on $\Lambda$; consequently we shall identify $V$ and $B$ with their images under this representation and define the uniform norm on $B$ by: $\|f\|=\sup \{|\bar{f}(\lambda)|: \lambda \in \Lambda\}=\sup \{|\lambda(f)|: \lambda \in \Lambda\}$ for $f \in B$. Condition (4) assures us that $\|f\|$ is finite and that $B$ is sufficiently large. It should be remarked that this representation is only a vehicle and will be discarded as soon as the desired function space appears.

3. The integral. We now focus our attention on conditions (1) and (2). Let $V^{+}=\{x \in V: x \geqq 0\}$.

LEMMA 2. Let $\nu: V^{+} \rightarrow R$ be a positive additive functional. Then there is a unique positive linear functional $\mu: V \rightarrow R$ agreeing with $v$ on $V^{+}$.

Proof. See Bourbaki [4, p. 34, Proposition 2]. 
Lemma 3. Let $V$ be a vector lattice with a semi-norm $p$. Then (1) and (2) hold if and only if there is a positive linear functional $\mu$ on $V$ such that $p(x)=\mu(|x|)$, for all $x \in V$. Moreover, if (1) and (2) hold, $\mu$ is uniquely determined.

Proof. Sufficiency. In any vector lattice we have the identities:

$$
\begin{array}{lrl}
|x+y| & =|x|+|y| & \text { for } x, y \geqq 0 . \\
|(|x|)| & =|x| & \text { for all } x \in V .
\end{array}
$$

The additivity of $\mu$ together with the assumption $p(x)=\mu(|x|)$ easily imply (1) and (2).

Necessity and uniqueness. Condition (1) says that $p$ is additive on the positive cone $V^{+}$. As in the proof of Lemma 2, we define $\mu(x)$ $=p\left(x^{+}\right)-p\left(x^{-}\right)$. Clearly $p(x)=p(|x|)=\mu(|x|)$. Q.E.D.

4. Representation of $B$. The fact that $B$ is a pointwise algebra of bounded functions (Lemma 1) enables us to adjoin an order unit $e$ (the constant function 1) to $B$ (for definition of an order unit see Kadison [5, p. 3]). Thus for any function $f$ in $B$ we can find an $\alpha \geqq 0$ such that $|f| \leqq \alpha e$. Let $B_{e}$ denote the algebra resulting from the adjunction of $e$ to $B$. Being a function lattice, $B_{e}$ is manifestly Archimedean in Kadison's sense and the "natural norm" $\|f\|$ $=\inf \{\alpha:|f| \leqq \alpha e\}$ is just the uniform norm inherited from $m(\Lambda)$. By condition (4), $B$ is complete in the uniform norm as is $B_{e}$.

We now appeal to a classical representation theorem for vector lattices.

THEOREM 2. Let $L$ be an Archimedean vector lattice with order unit e. If $L$ is complete in the norm $\|f\|=\inf \{\alpha:|f| \leqq \alpha e\}$ then one can find a compact Hausdorff space $S$ and a linear lattice isometry of $L$ with $C(S)$.

For the proof, we refer to $[2$, p. 103 , Theorem 3$]$ or to $[5$, p. 10 , Theorem 4.1 and Historical Remarks].

COROLlARY. $B$ is linearly, latticially and isometrically isomorphic to $C_{\infty}(E)$, the Banach algebra of all continuous functions on $E=S-\{\infty\}$ (locally compact Hausdorf) vanishing at $\infty$.

We may now identify $B$ with $C_{\infty}(E)$ and observe that the positive linear functional $\mu$ obtained in $\$ 3$ is a positive Radon measure on $E$ in the sense of Bourbaki [4]. By (4), $B=C_{\infty}(E)$ is dense in $V$ under the $p$-topology. But the ring of continuous functions with compact supports on $E$ is uniformly dense in $C_{\infty}(E)$ and it is easily seen that the former has $V$ as its completion under the $p$-topology. Thus $V$ is linearly, latticially and isometrically isomorphic to $\mathscr{L}^{1}(E, \mu)$. Clearly 
$V / p^{-1}(0)$ is abstractly identical with $L^{1}(E, \mu)$. This completes the proof of the main theorem.

5. The compact case. We now consider vector lattices $V$ satisfying $(1),(2)$ and (3) in the definition of ( $(\&)$-space and the additional conditions:

(4') $V$ is boundedly $\sigma$-complete, i.e., each countable set in $V$ which is bounded above has a least upper bound.

$\left(4^{\prime \prime}\right)$ There is a total family $\Lambda$ of linear lattice functionals such that the subspace $B=\left\{x \in V:\|x\|=\sup _{\lambda \in \Lambda}|\lambda(x)|<\infty\right\}$ is dense in $V$ under the $p$-topology and contains an element $e$ satisfying $\lambda(e)=1$, for each $\lambda \in \Lambda$.

THEOREM 3. Let $V$ be a vector lattice satisfying conditions (1)-(3), $\left(4^{\prime}\right)$ and $\left(4^{\prime \prime}\right)$. Then there is a compact Hausdorff space $S$ and a unique positive Radon measure $\mu$ on $S$ satisfying $\mu(e)=1$ such that $V$ is linearly, latticially and isometrically isomorphic to $\mathfrak{L}^{1}(S, \mu)$. Moreover, $V / p^{-1}(0)$ is abstractly identical with $L^{1}(S, \mu)$.

The proof proceeds much like the proof of the main theorem, but some additional facts are needed. The element $e$ in $\left(4^{\prime \prime}\right)$ will of course play the role of the constant function 1 . As in $\$ 3$, we obtain a unique positive linear functional $\mu$ on $V$ satisfying $p(x)=\mu(|x|)$. If we set $\mu_{1}(x)=(1 / \mu(e)) \cdot \mu(x)$, then $\mu_{1}$ is again a positive linear functional "normalized" by the condition $\mu_{1}(e)=1$. The semi-norm $p_{1}(x)$ $=\mu_{1}(|x|)$ associated with $\mu_{1}$ is evidently equivalent to the original semi-norm $p$.

As in $\S 2$, we regard $B$ as a function lattice.

Lemma 4. Let $V$ be a boundedly complete (resp. $\sigma$-complete) vector lattice, $B$ a lattice ideal in $V$. Then $B$ is boundedly complete (resp. $\sigma$-complete).

Proof. By Bourbaki [4, p. 21, Proposition 1], it suffices to show that any set $H$ of positive elements, directed by $\leqq$ and bounded above has a least upper bound. By assumption, sup $H$ exists in $V$ and $0 \leqq$ sup $H \leqq b$, where $b \geqq H$ is the element bounding $H$. Thus sup $H \in B$ by the lattice ideal property. For the $\sigma$-complete case take $H$ countable. Q.E.D.

Thus $B$ is a boundedly $\sigma$-complete algebra of bounded functions containing the order unit $e$ and normed by $\|f\|=\inf \{\alpha:|f| \leqq \alpha e\}$.

Lemma 5. $B$ is complete in the uniform norm.

Proof. Let $\left\{f_{i}\right\}$ be a Cauchy sequence in $B$. Then given $\epsilon>0$ there exists an integer $k$ such that $\left\|f_{n}-f_{m}\right\|<\alpha$, for $m, n \geqq k$ and $\alpha=\epsilon / 2$. 
Now $\left|f_{n}-f_{k}\right| \leqq \alpha e$ so $-\alpha e \leqq f_{n}-f_{k} \leqq \alpha e$ and $f_{k}-\alpha e \leqq f_{n} \leqq f_{k}+\alpha e$. Hence $f_{n}$ is bounded above and below in $B$. Setting $f=\lim _{\sup _{n \rightarrow \infty}} f_{n}$ we have $f \in B$ by Lemmas 1 and 4 and condition $\left(4^{\prime}\right)$; moreover $f_{k}-\alpha e \leqq f$ $\leqq f_{k}+\alpha e$ so $\left|f-f_{k}\right| \leqq \alpha e$ and $\left\|f-f_{k}\right\| \leqq \alpha=\epsilon / 2<\epsilon$. Thus $\left\|f-f_{i}\right\| \rightarrow 0$ as $i \rightarrow \infty$. Q.E.D.

By Theorem 2, $B$ is linearly, latticially and isometrically isomorphic to $C(S)$ for some compact Hausdorff space $S$ in such a way that $e$ corresponds to the constant function 1 on $S$. $V$ is then abstractly identical with $\mathscr{L}^{1}\left(S, \mu_{1}\right)$ and $V / p_{1}^{-1}(0)$ can be identified with $L^{1}\left(S, \mu_{1}\right)$. This completes the proof of Theorem 3.

The example cited in $\$ 1$ seems to suggest that the locally compact Hausdorff space $E$ obtained via the representation is closely related to Segal's "perfection" of a localizable measure space (see [6]).

The author is grateful to Professors F. D. Quigley and F. B. Wright for their suggestions and encouragement.

\section{REFERENCES}

1. S. Kakutani, Concrete representation of abstract $(L)$-spaces and the mean ergodic theorem, Ann. of Math. vol. 42 (1941) pp. 523-537.

2. M. M. Day, Normed linear spaces, Ergebnisse der Mathematik, Neue Folge, Berlin, Springer, Heft 21, 1958.

3. T. Nakayama, Note on the lattice-ordered groups, Proc. Imp. Acad. Tokyo vol. 18 (1942) pp. 1-4.

4. N. Bourbaki, Integration, Chapters I-IV, Paris, Hermann and Cie., 1952.

5. R. V. Kadison, $A$ representation theory for commutative topological algebra, Memoirs Amer. Math. Soc. no. 7 (1951).

6. I. E. Segal, Equivalences of measure spaces, Amer. J. Math. vol. 73 (1951) pp. 275-313.

TUlane University 